\title{
Forças de vínculo no caso holônomo
}

\author{
(Forces of constraint in the holonomic case)
}

\author{
Nivaldo A. Lemos 1 \\ Departamento de Física Universidade Federal Fluminense, Niterói, RJ, Brasil \\ Recebido em 20/02/04; Aceito em 25/06/04
}

\begin{abstract}
É possível, por meios simples e diretos, encontrar as forças de vínculo para sistemas holônomos empregando as equações de Lagrange mas sem o uso da técnica dos multiplicadores de Lagrange. O método é descrito em sua generalidade e sua eficácia é demonstrada com a ajuda de alguns exemplos típicos.
\end{abstract}

Palavras-chave: dinâmica lagrangiana, forças de vínculo.

It is possible, by simple and direct means, to find the forces of constraint for holonomic systems employing Lagrange's equations but without the use of the Lagrange multiplier technique. The method is described in its generality, and its effectiveness is demonstrated with the help of a few typical examples.

Keywords: Lagrangian dynamics, forces of constraint.

\section{Introdução}

Os textos clássicos de mecânica analítica mostram como o método dos multiplicadores de Lagrange incorpora uma extensa classe de vínculos não-holônomos ao escopo do formalismo lagrangiano, ressaltam que as forças de vínculo emergem automaticamente como um valioso subproduto da técnica e costumam notar, em seguida, que o método também funciona quando os vínculos são holônomos [1]. Às vezes, o método é introduzido primordialmente para lidar com vínculos holônomos, e somente mais tarde assinala-se que ele também abrange certos vínculos não-holônomos [2]. Qualquer que seja o caminho seguido, permanece o fato de que, usualmente, os exemplos escolhidos para ilustrar o método são sistemas sujeitos a vínculos holônomos [1,2]. Isto deixa o estudante com a falsa impressão de que, na dinâmica lagrangiana, um apelo a multiplicadores de Lagrange é inescapável caso se esteja interessado em obter as forças de vínculo. $\mathrm{O}$ propósito desta nota é chamar a atenção para o fato de que, no caso holônomo, as forças de vínculo podem ser obtidas de forma simples e direta sem recorrer a multiplicadores de espécie alguma.
A dedução das equações de Lagrange para sistemas holônomos contendo $N$ partículas começa decompondo a força sobre a $i$-ésima partícula como

$$
\mathbf{F}_{i}=\mathbf{F}_{i}^{(a)}+\mathbf{f}_{i}
$$

onde $\mathbf{F}_{i}^{(a)}$ é a força aplicada e $\mathbf{f}_{i}$ é a força de vínculo. De modo geral, as forças aplicadas é que devem ser consideradas as verdadeiras causas do movimento, as forças de vínculo servindo meramente para assegurar a preservação das restrições geométricas ou cinemáticas no decurso do tempo. A hipótese - verdadeira na quase totalidade das situações de interesse físico - de que o trabalho virtual total das forças de vínculo é zero conduz ao princípio de d'Alembert

$$
\sum_{i}\left(\dot{\mathbf{p}}_{i}-\mathbf{F}_{i}^{(a)}\right) \cdot \delta \mathbf{r}_{i}=0
$$

no qual as forças de vínculo não mais aparecem (os $\delta \mathbf{r}_{i}$ são deslocamentos virtuais).

Quando os vínculos são holônomos, existem coordenadas generalizadas $q_{1}, \ldots, q_{n}$ tais que

$$
\mathbf{r}_{i}=\mathbf{r}_{i}\left(q_{1}, \ldots, q_{n}, t\right), i=1, \ldots, N,
$$

e as equações de vínculo são identicamente satisfeitas. Se as forças aplicadas são dedutíveis de um potencial

\footnotetext{
${ }^{1}$ Enviar correspondência para Nivaldo A. Lemos. E-mail: nivaldo@if.uff.br.
} 
escalar $V\left(\mathbf{r}_{1}, \ldots, \mathbf{r}_{N}, t\right)$, isto é,

$$
\mathbf{F}_{i}^{(a)}=-\nabla_{i} V=-\left(\frac{\partial V}{\partial x_{i}} \hat{\mathbf{i}}+\frac{\partial V}{\partial y_{i}} \hat{\mathbf{j}}+\frac{\partial V}{\partial z_{i}} \hat{\mathbf{k}}\right),
$$

argumentos bem conhecidos [1] levam do princípio de d'Alembert às equações de Lagrange

$$
\frac{d}{d t}\left(\frac{\partial L}{\partial \dot{q}_{k}}\right)-\frac{\partial L}{\partial q_{k}}=0 \quad, \quad k=1, \ldots, n .
$$

A lagrangiana é dada por $L=T-V$, onde $T$ é a energia cinética total do sistema. As forças de vínculo não aparecem nas equações de Lagrange porque o potencial $V$ refere-se apenas às forças aplicadas, e esta é uma das grandes virtudes do formalismo lagrangiano.

Caso estejamos interessados nas forças de vínculo, devemos retornar à formulação newtoniana. Introduzindo a decomposição (1) nas equações de movimento de Newton

$$
m_{i} \ddot{\mathbf{r}}_{i}=\mathbf{F}_{i} \quad, \quad i=1, \ldots, N
$$

obtém-se

$$
\mathbf{f}_{i}=m_{i} \ddot{\mathbf{r}}_{i}-\mathbf{F}_{i}^{(a)}
$$

De acordo com esta equação, as forças de vínculo podem ser determinadas adotando o seguinte procedimento simples: (i) calcule a aceleração $\ddot{\mathbf{r}}_{i}$ tomando a segunda derivada de (3); (ii) elimine as acelerações generalizadas $\ddot{q}_{k}$ por meio das equações de Lagrange (5); (iii) calcule $\mathbf{F}_{i}^{(a)}$ por meio de (4); (iv) insira os resultados dos passos anteriores em (7) para obter as forças de vínculo.

Exemplo 1. Como primeiro e venerável exemplo, consideremos a máquina de Atwood, que consiste em duas massas unidas por um fio leve e inextensível passando por uma polia de massa desprezível (ver Fig. 1). Escolhendo um eixo vertical $x$ orientado para baixo, a energia cinética é

$$
T=\frac{m_{1}}{2} \dot{x}_{1}^{2}+\frac{m_{2}}{2} \dot{x}_{2}^{2},
$$

e o potencial das forças aplicadas é

$$
V=-m_{1} g x_{1}-m_{2} g x_{2} .
$$

O vínculo holônomo

$$
x_{1}+x_{2}=\ell,
$$

onde $\ell$ é uma constante, nos permite tomar $x_{1}$ como coordenada generalizada. A lagrangiana torna-se

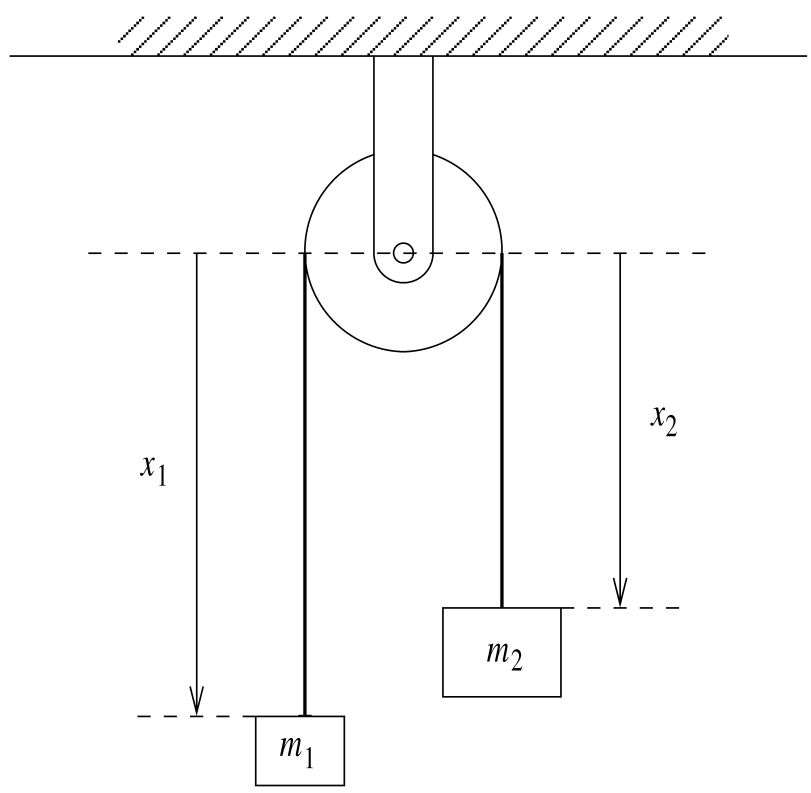

Figura 1 - Máquina de Atwood.

$$
L=\frac{m_{1}+m_{2}}{2} \dot{x}_{1}^{2}+\left(m_{1}-m_{2}\right) g x_{1}+m_{2} g \ell,
$$

da qual resulta a única equação de Lagrange:

$$
\begin{gathered}
\frac{d}{d t}\left(\frac{\partial L}{\partial \dot{x}_{1}}\right)-\frac{\partial L}{\partial x_{1}}=0 \Longrightarrow\left(m_{1}+m_{2}\right) \ddot{x}_{1}= \\
\left(m_{1}-m_{2}\right) g .
\end{gathered}
$$

A fim de encontrar a tensão no fio, executemos as duas primeiras instruções de nossa receita. A aceleração da primeira massa é

$$
\ddot{\mathbf{r}}_{1}=\ddot{x}_{1} \hat{\mathbf{i}}=\frac{m_{1}-m_{2}}{m_{1}+m_{2}} g \hat{\mathbf{i}},
$$

onde a equação de Lagrange (12) foi usada ( $\hat{\mathbf{i}}$ é o vetor unitário apontando verticalmente para baixo). Como terceiro passo, escrevemos $\mathbf{F}_{1}^{(a)}=-\nabla_{1} V=m_{1} g \hat{\mathbf{i}}$, que é o peso da primeira massa. Finalmente, introduzindo esses resultados em (7) encontramos o resultado bem conhecido

$$
\mathbf{f}_{1}=m_{1} \ddot{\mathbf{r}}_{1}-m_{1} g \hat{\mathbf{i}}=-\frac{2 m_{1} m_{2}}{m_{1}+m_{2}} g \hat{\mathbf{i}},
$$

o sinal negativo indicando que a tensão no fio aponta verticalmente para cima.

Exemplo 2. Uma conta desliza sem atrito ao longo de uma fio rígido que gira uniformemente num plano no espaço vazio (ou num plano horizontal próximo à 
superfície da Terra). Se $r$ é a distância da conta ao eixo de rotação, as coordenadas cartesianas da conta no plano do movimento são dadas por

$$
x=r \cos \omega t \quad, \quad y=r \operatorname{sen} \omega t,
$$

onde $\omega$ é a velocidade angular constante de rotação. Não há forças aplicadas, de modo que a lagrangiana é

$$
L=T=\frac{m}{2}\left(\dot{x}^{2}+\dot{y}^{2}\right)=\frac{m}{2}\left(\dot{r}^{2}+\omega^{2} r^{2}\right),
$$

e a única equação de Lagrange é

$$
\frac{d}{d t}\left(\frac{\partial L}{\partial \dot{r}}\right)-\frac{\partial L}{\partial r}=0 \Longrightarrow \ddot{r}=\omega^{2} r
$$

As componentes cartesianas da aceleração da conta são

$$
\begin{aligned}
\ddot{x} & =\frac{d^{2}}{d t^{2}}(r \cos \omega t) \\
& =\ddot{r} \cos \omega t-2 \omega \dot{r} \operatorname{sen} \omega t-\omega^{2} r \cos \omega t \\
& =-2 \omega \dot{r} \operatorname{sen} \omega t, \\
\ddot{y} & =\frac{d^{2}}{d t^{2}}(r \operatorname{sen} \omega t) \\
& =\ddot{r} \operatorname{sen} \omega t+2 \omega \dot{r} \cos \omega t-\omega^{2} r \operatorname{sen} \omega t \\
& =2 \omega \dot{r} \cos \omega t,
\end{aligned}
$$

tendo sido usada a Eq. 177. Como não há forças aplicadas, a Eq. (7) fornece

$$
\begin{gathered}
\mathbf{f}=m \ddot{\mathbf{r}}=-2 m \omega \dot{r} \operatorname{sen} \omega t \hat{\mathbf{i}}+2 m \omega \dot{r} \cos \omega t \hat{\mathbf{j}}= \\
-2 m \omega \dot{r} \hat{\mathbf{e}}_{\theta},
\end{gathered}
$$

onde $\hat{\mathbf{e}}_{\theta}=-\operatorname{sen} \omega t \hat{\mathbf{i}}+\cos \omega t \hat{\mathbf{j}}$ é o vetor unitário perpendicular ao fio, pois $\theta=\omega t$. A força de vínculo é perpendicular ao fio, resultado esperado dada a ausência de atrito de deslizamento.

Exemplo 3. Como terceiro e último exemplo, consideremos um cilindro de raio $a$ que rola sem deslizar sobre um cilindro fixo de raio $b$, problema que encontra-se resolvido detalhadamente em [2] pelo método dos multiplicadores de Lagrange. Sejam $x, y$ coordenadas cartesianas com origem no centro do cilindro fixo: o eixo $x$ é horizontal e o eixo $y$ é vertical orientado para cima. Seja $\phi$ o ângulo de rotação do cilindro móvel em torno do seu eixo de simetria. É conveniente introduzir coordenadas polares $r$ e $\theta$ do centro do cilindro móvel, $\operatorname{com} \theta$ contado a partir do eixo $y$ (vide Fig. 2). A energia cinética do cilindro móvel compõe-se da energia cinética do centro de massa e da energia de rotação em torno do centro de massa, isto é,

$$
T=\frac{m}{2}\left(\dot{r}^{2}+r^{2} \dot{\theta}^{2}\right)+\frac{1}{2} I \dot{\phi}^{2},
$$

onde $I=m a^{2} / 2$ é o momento de inércia do cilindro em torno do seu eixo de simetria. A energia potencial é $V=m g y=m g r \cos \theta$, de modo que a lagrangiana escreve-se

$$
L=\frac{m}{2}\left(\dot{r}^{2}+r^{2} \dot{\theta}^{2}\right)+\frac{m a^{2}}{4} \dot{\phi}^{2}-m g r \cos \theta .
$$

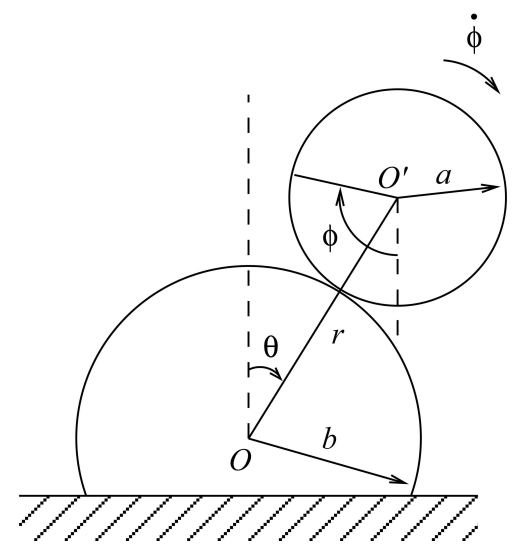

Figura 2 - Cilindro rolando sem deslizar sobre cilindro fixo.

Neste problema há dois vínculos holônomos, a saber:

$$
r \dot{\theta}=a \dot{\phi}
$$

(vínculo de rolar sem deslizar) ;

$$
r=a+b \quad \text { (vínculo de contato) . }
$$

O vínculo de rolar sem deslizar exprime a exigência de que o centro de massa do cilindro mova-se com velocidade $v_{\theta}=r \dot{\theta}$ igual àquela gerada por sua rotação com velocidade angular $\dot{\phi}$ em torno do eixo instantâneo de rotação, que passa pelo ponto de contato entre os cilindros. Substituindo (23) e (24) em (22) resulta a lagrangiana reduzida

$$
L=\frac{3 m}{4}(a+b)^{2} \dot{\theta}^{2}-m g(a+b) \cos \theta,
$$

da qual decorre a equação de Lagrange

$$
\frac{3 m}{2}(a+b)^{2} \ddot{\theta}-m g(a+b) \operatorname{sen} \theta=0 \text {. }
$$


As componentes cartesianas da aceleração do centro de massa do cilindro são

$$
\begin{aligned}
\ddot{x} & =\frac{d^{2}}{d t^{2}}[(a+b) \operatorname{sen} \theta] \\
& =(a+b) \ddot{\theta} \cos \theta-(a+b) \dot{\theta}^{2} \operatorname{sen} \theta \\
& =\frac{2 g}{3} \operatorname{sen} \theta \cos \theta-(a+b) \dot{\theta}^{2} \operatorname{sen} \theta, \\
\ddot{y} & =\frac{d^{2}}{d t^{2}}[(a+b) \cos \theta] \\
& =-(a+b) \ddot{\theta} \operatorname{sen} \theta-(a+b) \dot{\theta}^{2} \cos \theta \\
& =-\frac{2 g}{3} \operatorname{sen}^{2} \theta-(a+b) \dot{\theta}^{2} \cos \theta,
\end{aligned}
$$

onde a Eq. (26) foi usada.

A força aplicada é $\mathbf{F}^{(a)}=-m g \hat{\mathbf{j}}$, de modo que a força de vínculo sobre o cilindro móvel é

$\mathbf{f}=m \ddot{\mathbf{r}}-\mathbf{F}^{(a)}=\left[\frac{2 m g}{3} \operatorname{sen} \theta \cos \theta-m(a+b) \dot{\theta}^{2} \operatorname{sen} \theta\right] \hat{\mathbf{i}}-$

$$
\left[\frac{2 m g}{3} \operatorname{sen}^{2} \theta+m(a+b) \dot{\theta}^{2} \cos \theta-m g\right] \hat{\mathbf{j}} .
$$

Em particular, a força de reação normal do cilindro fixo sobre o cilindro móvel é a componente radial de f, isto é,

$$
\begin{gathered}
f_{r}=\mathbf{f} \cdot \hat{\mathbf{e}}_{r}=\mathbf{f} \cdot(\operatorname{sen} \theta \hat{\mathbf{i}}+\cos \theta \hat{\mathbf{j}})= \\
m g \cos \theta-m(a+b) \dot{\theta}^{2} .
\end{gathered}
$$

Comparado com o tratamento tradicional deste problema pela técnica dos multiplicadores de Lagrange [2], o presente método revela-se mais conciso e direto.

Outra vantagem geral do método é que o vetor força de vínculo sobre cada partícula é obtido diretamente, sem passar primeiro pelas determinação de suas componentes generalizadas em termos dos multiplicadores de Lagrange. O método é limitado, contudo, pois só é aplicável quando todos os vínculos são holônomos.

\section{Referências}

[1] H. Goldstein, Classical Mechanics (Addison-Wesley, Reading, MA, 1980), $2^{a} \underline{a}$ edição.

[2] A.L. Fetter e J.D. Walecka, Theoretical Mechanics of Particles and Continua (McGraw-Hill, New York, 1980). 